\title{
Testing and Applications of Non-Linear Wave Shaping Circuits Based on Zener Diodes at Cryogenic Temperatures
}

\author{
A. M. Abd El-Maksood \\ Electronics Engineering Department, Exploration Sector,Nuclear Materials Authority, P.O.Box 530, Maadi- \\ 11728,Maadi, Cairo, Egypt \\ E-mail: ashrafmosleh11@gmail.com
}

\begin{abstract}
Applications of wave-shaping clipping circuits based on Zener diodes are of great interest in a wide range of modern electronic systems. As well, given the strong interest in space research and trips to distant planets, where the journey takes long periods. Therefore, the matter requires reliance on electronic systems with special specifications commensurate with the nature of the extremely low temperature environments, down to cryogenic level (around $90 \mathrm{~K}$ ). So, the present paper was concerned with studying the stability of the performance of different non-linear wave shaping systems, based on silicon Zener diodes, whenever operates at very low temperatures down to cryogenic levels. From which, it is clear that, for BZX79-C4V7 and BZX79-C5V6 Zeners, such electronic systems were shown to be un-sensitive to temperature variations. On the other hand, low breakdown voltage Zeners (BZV86-1V4 and BZX83-C3V6), the clipping edges were shown to be increases with lowering temperatures from $300 \mathrm{~K}$ down to $93 \mathrm{~K}$. Finally, for Zener diodes with $\mathrm{V}_{Z}$ greater than 6.0 V (BZX83-C6V8 and BZX55C9V1), the temperature coefficient is positive, so the clipping edges decreases with lowering temperatures, for the same range of temperatures.
\end{abstract}

\section{Keywords}

Nonlinear wave shaping clipping circuits, Zener diodes, breakdown, wave shaping, voltage, environment, shunt Zener diode clippers and cryogen temperatures.

\section{INTRODUCTION}

Two important classes of circuits known as non-linear wave shaping circuits are clipping - and clamping - circuits. Noting that, clamping operation involves storage of energy in the circuit, while, energy storage has no role to play in clipping circuits [1]. In general, a clipper circuit is a circuit which cuts off a portion above a predetermined level and /or below a predetermined level of a voltage signal to be inputted, using nonlinear element such as a diode [2]. Such a clipper circuit has been used in a variety of ways in the electronic circuit field. For example, the clipper circuit has been frequently adapted when a stable voltage is required to be supplied to an associated circuit in a Switched-Mode Power Supply (SMPS) circuit, the noise included in a received voice signal in a field of the communication should be eliminated, or a rectangular waveform is formed using a received voice signal. Also, clipping circuits are known as limiters, amplitude selectors, or slicers [3]. Such circuits are used to prevent breakdown of active devices by limiting maximum voltage that the active device may be subjected to. They are also used at the input terminals of Op-Amp's to limit maximum differential voltage [4]. A clipper is employed as an entrance protective circuit to avoid voltage peaks [5]. In this concern, there are two general categories of clippers: series and parallel (shunt) [3]. Voltage limiters (clippers) may be divided into two classes: common with $p-n$ junction on a single crystal and micro assemblies made of two or three crystals. Single-crystal voltage limiters are used for protecting direct current circuitry from emergency pulses. Alternating current circuits are protected with two back-to-back connected limiters or dual-crystal limiters obtained by unifying two diodes with direct or reverse $p-n$ junctions [6].

\section{THEORY of OPERATION for SHUNT ZENER DIODE CLIPPERS}

Shunt Zener diode clippers are the simplest form of such networks, where it is basically consists of single Zener diode connected parallel to the load. In this concern, Figure 1 shows the three versions of such clipper/limiter circuits, i.e., positive (Figure 1a) -negative (Figure 1b) - and - double ended (Figure 1c) - clippers/limiters. For the operation of the first one (Figure 1a), positive clipper, normally, it removes some part of waveform (from an ac input) in positive half cycle. The input waveform is limited, resulting in the output waveform from the negative Zener rated voltage to +0.70 Volt. On the other hand, the negative clipper (Figure 1b) removes some part of waveform (from an ac input) in negative half cycle. In this circuit the output voltage levels will be limited to between - 0.70 Volt and the Zener voltage rating [7]. Finally, the double ended clipper circuits (Figure 1c) are generally well-known in the prior art and one such clipping circuit [8]. In this case, the circuit removes some part of waveform (from an ac input) in both positive and negative half cycles. The two Zener diodes $D_{1}$ and $D_{2}$ in the circuit have breakdown voltage $\left(V_{Z_{1}}\right)$ and $\left(V_{Z 2}\right)$, respectively [4]. 


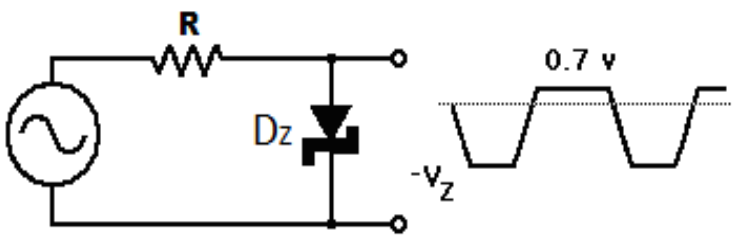

(a)

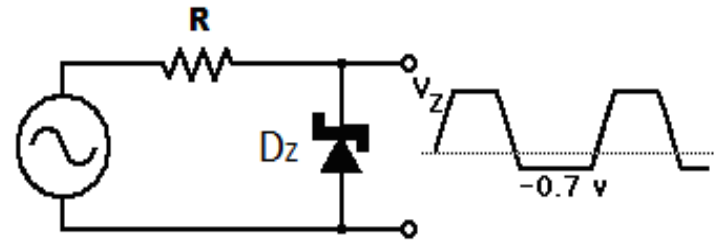

(b)

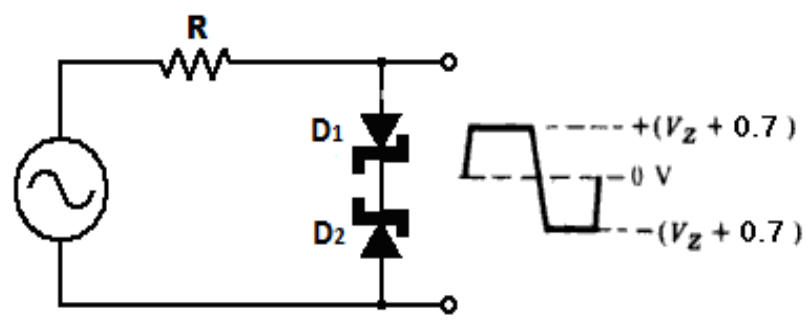

(c)

Fig 1: Shunt Zener diode clippers (a) Positive, (b) negative, and (c) double ended.

\section{EXPERIMENTAL PROCEDURES}

During the course of the study, the operation, applications and the effects of temperature down to very low levels (cryogenic) on shunt Zener diode clippers, operate in their three modes, were investigated. In this concern, the study is based on different Zener diodes covering wide range of low breakdown voltages range from 1.40 Volts up to 9.10 Volts (BZV86-1V4, BZX83-C3V6, BZX79-C4V7, BZX79-C5V6, BZX83-C6V8 and BZX55C9V1). Figure 1 shows the proposed clipper circuits, where, the input signal was a 9.0 Volts sine wave pulse with frequency of $400 \mathrm{~Hz}$. Finally, all measurements were carried out at different temperature levels within the range from room level (300 K) down to cryogenic level $(93 \mathrm{~K})$ using the cooling system, previously published by the authors [9]. A temperature rate of change of $10^{\circ} \mathrm{C} / \mathrm{min}$ was used, and a soak time of at least 20 minutes was allowed at every test temperature.

The basic cooling system components are: a special glass cell with its glass cover having embedded tungeston electrodes, pressurized gas cylinder and liquid nitrogen dewar flask (Figure 2). The glass container was connected to a vacuum system and to the nitrogen gas cylinder via three way T-junction glass valve. The glass cell immersed in the dewar flask, and the open part of the top of the dewar flask was covered by thin disc of insulating material for thermal isolation. The glass cell was evacuated by a rotary pump and hence the dry nitrogen gas was admitted to the cell and its pressure is then adjusted to be slightly higher than the atmospheric pressure to ensure the absence of any humidity from the atmosphere inside the cell. The specimen temperature could be decreased by the addition of liquid nitrogen to the dewar flask.

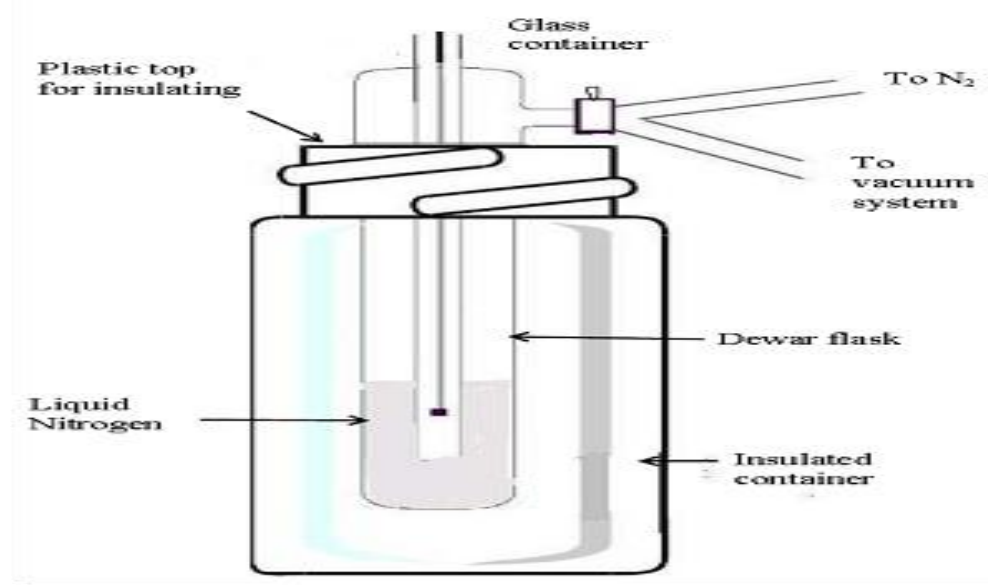

Fig 2: The construction of cooling system used for controlling the sample temperature from room temperature down to liquid nitrogen temperature. 


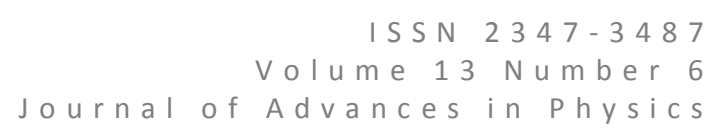

\section{RESULTS AND DISCUSSIONS}

\subsection{Temperature Dependence of Zener Diodes Characteristics}

Temperature variations can have significant effects on the electrical characteristics of semiconductor diodes, the matter which is clearly shown from Figures (3 and 4) for the case of the proposed different Zener diodes (BZV86-1V4, BZX83C3V6, BZX79-C4V7, BZX79-C5V6, BZX83-C6V8 and BZX55C9V1). From which, it is clear that the forward threshold voltage decreases with increasing temperature. As an example, for the diode BZV86-1V4, the threshold voltage had a value of $\sim 0.82$ Volt, measured at room temperature $(300 \mathrm{~K})$, goes to $\sim 1.07$ Volts with cooling the diode down to $93 \mathrm{~K}$ for a given current. Similar behaviors were obtained for the remaining Zener diodes.

In general, Zener diodes are not used with forward bias, but if forward biased operation is requested, deliberate attention should be paid to the absolute maximum ratings $[10,11]$. The shown effects are mainly due to that the operation of ordinary diodes at cryogenic temperatures is mainly influenced by the effect of carrier freeze out at low temperatures. In addition to freeze out effect, the increase in the junction potential due to Fermi level shift, also, affects the operation of the diodes. The forward bias voltage drop $\mathrm{V}_{0}$ (threshold voltage $\mathrm{V}_{\gamma}$ ) can be a measure of the junction potential [12]. Over an intermediate range of temperatures in doped silicon, including room temperature, there is enough thermal energy to fully ionize all mobile carriers. This is due to the small energy gap between the impurity energy states and conduction band (or valence band for p-type). The mobile carrier concentration over this range is generally assumed to be the same as the doping concentration (Figure 4). As temperature decreases below this range, however, there may no longer be enough thermal energy to ionize all the impurity atoms. This condition is known as incomplete ionization, or freeze-out [13].

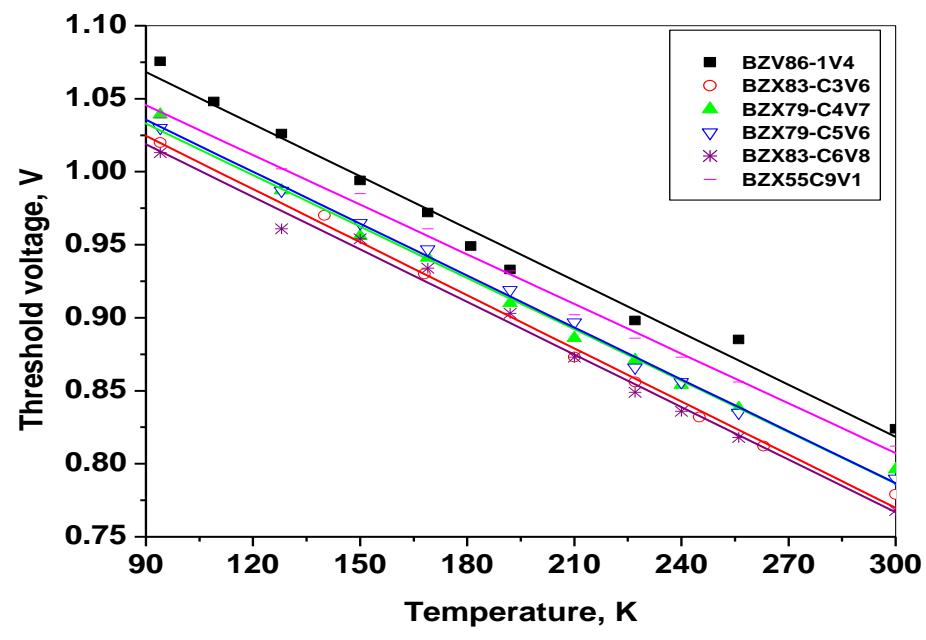

Fig 3: Temperature dependence of the forward threshold voltage for the proposed Zener diodes.

The breakdown Zener voltage $\left(V_{Z}\right)$ dependence on temperature, plotted at two different operating currents was shown in Figure 4. From which, it is to be noted that, the temperature dependence of $V_{Z}$ should be considered not only for the change in ambient temperature but also for the junction temperature increase due to the Zener diode power dissipation [10]. It is known that two mechanisms of breakdown can be present in Zener diodes: avalanche breakdown and Zener (tunneling) breakdown. For the silicon and germanium diodes, the breakdown is due to tunneling effect when the breakdown voltage is less than $V_{B r}^{T}=4 x E_{g}$ (Figure $4(\mathrm{a}, \mathrm{b})$ ) and is caused by avalanche multiplication when the breakdown voltages is in excess of $V_{B r}^{A}=6 x E_{g}$ (Figure $\left.4(\mathrm{e}, \mathrm{f})\right)$, where Eg is the semiconductor band gap in eV. For voltages between $V_{B r}^{T}$ and $V_{B r}^{A}$ both mechanisms of breakdown is present (Figure 4 (c, d)) [14]. 


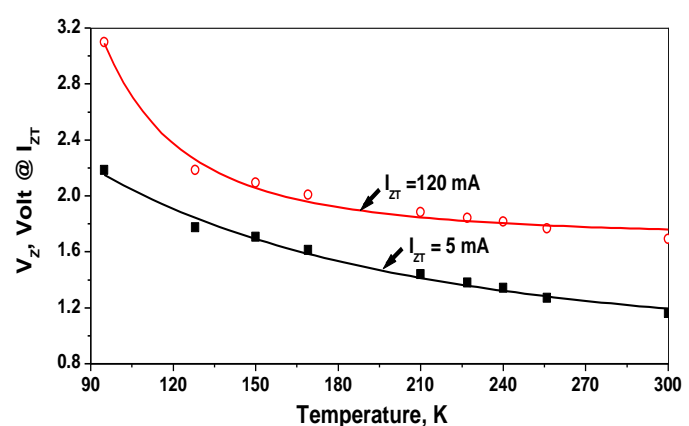

(a)

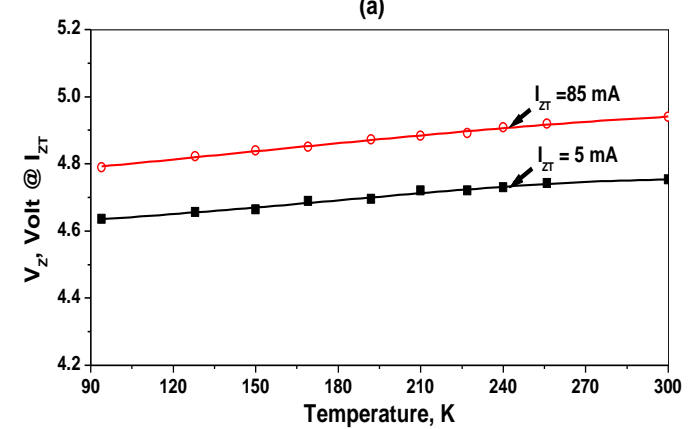

(c)

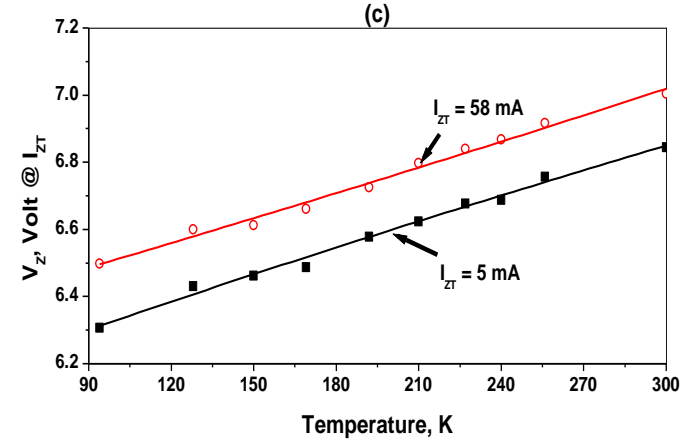

(e)

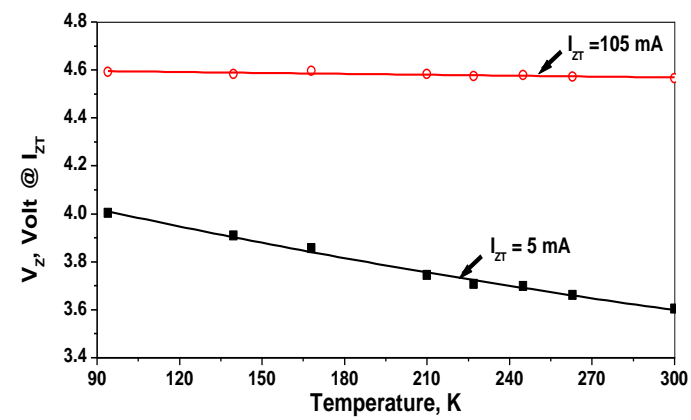

(b)

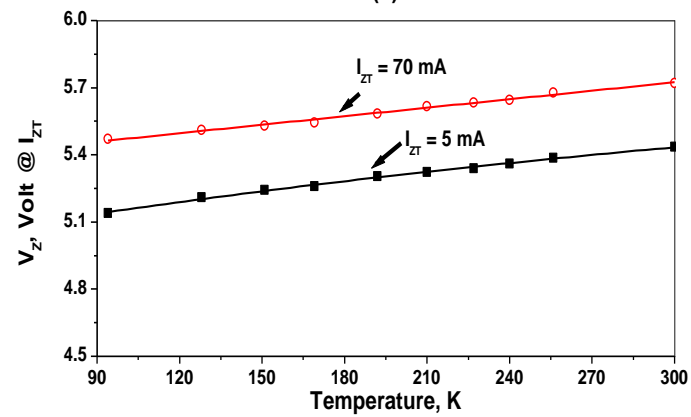

(d)

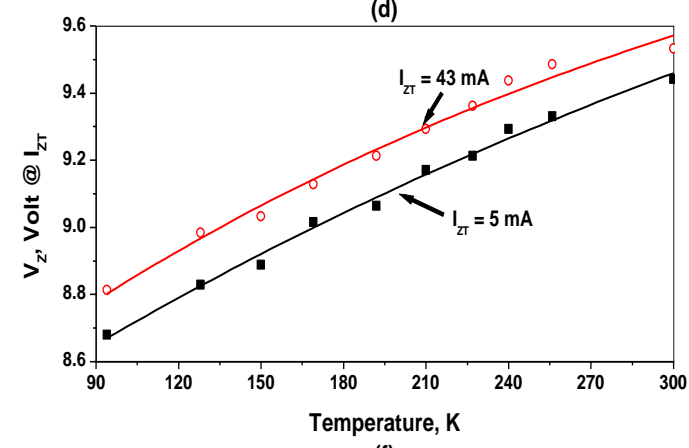

(f)

Fig 4: Temperature dependence of the breakdown Zener voltage at a given test current for the proposed Zener diode (a) BZV86-1V4, (b) BZX83-C3V6, (c) BZX79-C4V7, (d) BZX79-C5V6, (e) BZX83-C6V8, and (f) BZX55C9V1.

The temperature coefficient (TC) versus Zener voltage of the proposed diodes was shown in Figure 5. From which, it clearly shown that a negative to positive temperature coefficient "transition" will occur in the vicinity of the breakdown voltage value of 5.0 Volts for most Zener product designs. For Zener breakdown voltages value above 5.0 Volts, the positive TC for avalanche breakdown is domain. Finally, for those diodes with breakdowns around 5.0 Volts or below, where transition to field emission or "tunneling breakdown" occurs, negative values of TC were observed $[9,15]$. 


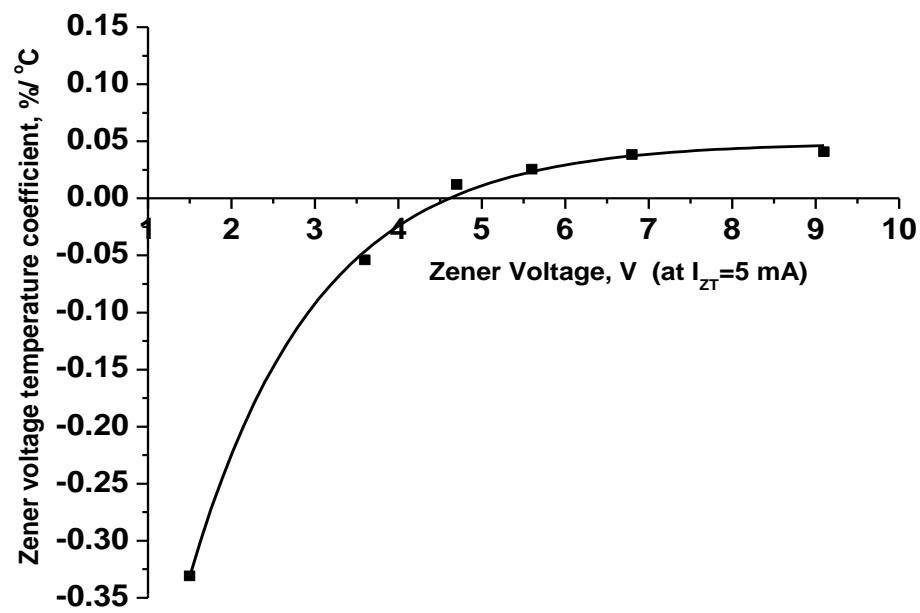

Fig 5: Temperature coefficient versus Zener voltage of the proposed six diodes.

\subsection{Performance of Shunt Zener Diode Clippers at Low Temperature}

\subsubsection{Positive Zener Diode Clippers}

The transfer characteristic graphs of the Zener diodes positive parallel clippers were shown in Figure 6. The graphs represent the figure of output voltage $\left(\mathrm{V}_{0}\right)$ against input $\left(\mathrm{V}_{\mathrm{i}}\right)$ with time as an implicit variable [16], for the studied Zener diodes, plotted at two temperature levels of $300 \mathrm{~K}$ and $93 \mathrm{~K}$ (for simplicity). The mathematical equation for such a figure is given by [1]:

$$
\begin{array}{lll}
V_{0}=-V_{Z} & \text { for } V_{i}<-V_{Z}, & D_{Z} \text { in breakdown region } \\
V_{0}=V_{i} & \text { for }-V_{Z}<V_{i}<V_{\gamma}, & D_{Z} \text { isopen } \\
V_{0}=V_{\gamma} & \text { for } V_{i}>V_{\gamma}, & D_{Z} \text { inconducts }
\end{array}
$$

During the positive half cycle of the 9.0 Volts peak input signal, the Zener diodes are forward biased, that is, conducting. The positive half cycle of the sine wave is shorted out. The positive half cycle of outputs waveforms would be clipped at 0 $\mathrm{V}$ for an ideal diode. The waveform is clipped at $\mathrm{V}_{\gamma}=+0.75 \mathrm{Volt}$ at room temperature $(300 \mathrm{~K})$, while at $93 \mathrm{~K}$ is clipped at about $\mathrm{V}_{\gamma}=+1.0$ Volt for BZV86-1V4 (Figure 6a), this increases in forward threshold voltage $\left(\mathrm{V}_{\gamma}\right)$ due to the effect of low temperature on forward characteristics (Figure 3). On the other hand, during the negative half cycle of the input signal, the Zener diodes are reversed biased. Hence, the clipper will limit the negative half cycle of a signal to its reverse breakdown voltages, - 1.40 Volts, measured at room temperature level $(300 \mathrm{~K})$, the value which was shown to be increased up to 2.0 Volts, measured at $93 \mathrm{~K}$ (Figure 6). This increase in the reverse breakdown voltages are due to the effect of low temperature on reverse characteristics of the Zener diode of type BZV86-1V4 (Figure 4a). Where, any change in junction temperature generally changes the Zener voltage $\mathrm{V}_{\mathrm{Z}}$. The temperature coefficient is approximately $+2 \mathrm{mV} /{ }^{\circ} \mathrm{C}[17]$. 


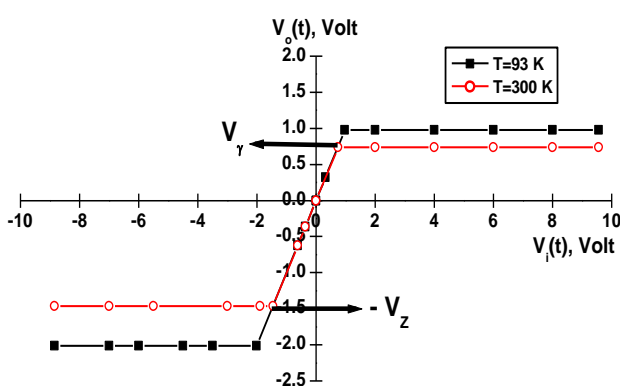

(a)

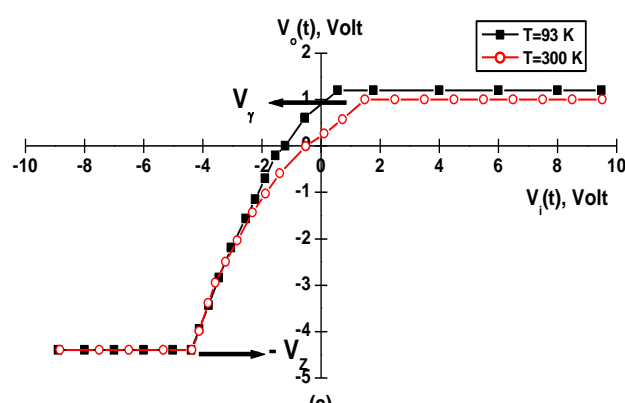

(c)

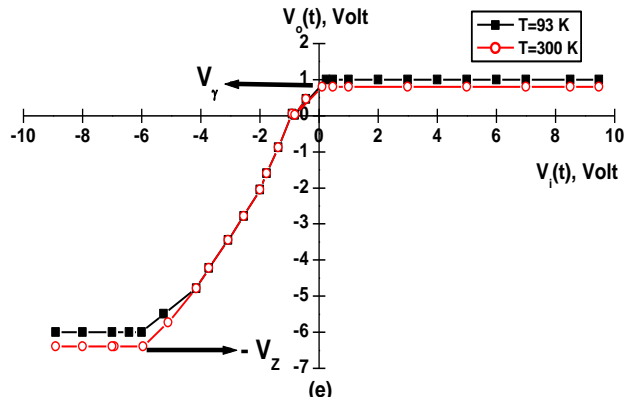

$-71$
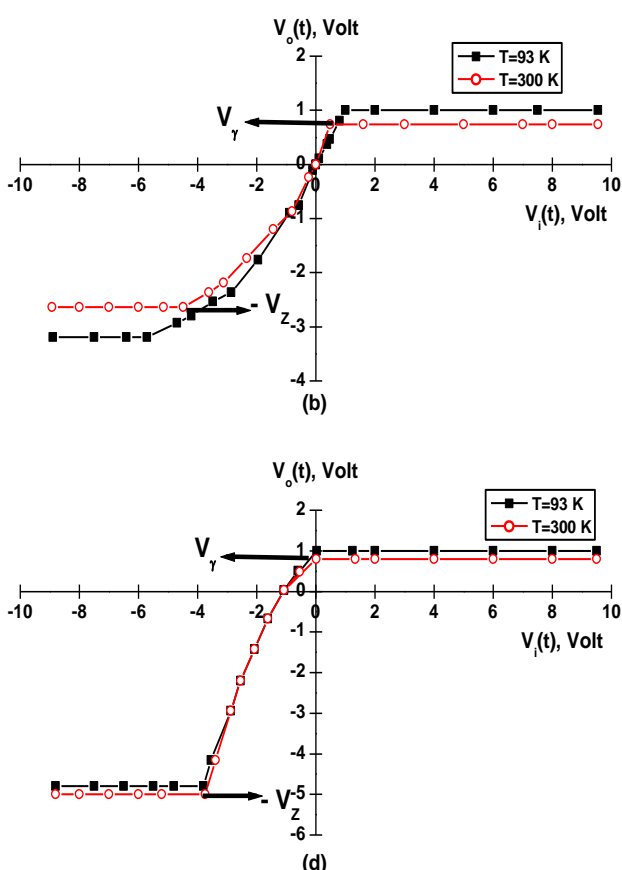

(d)

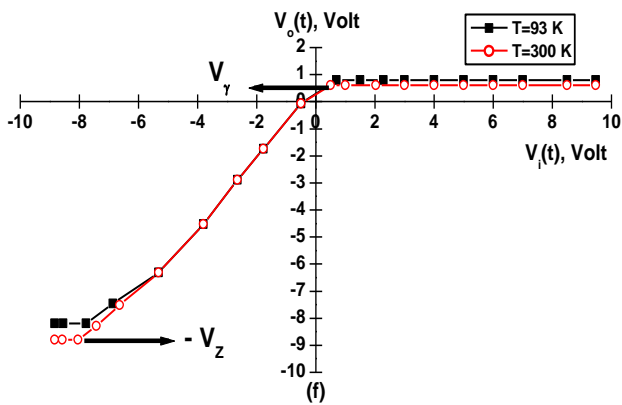

Fig 6: Transfer characteristics graphs of unsymmetrical positive clipper based on different Zener diodes (a) BZV86-1V4, (b) BZX83-C3V6, (c) BZX79-C4V7, (d) BZX79-C5V6, (e) BZX83-C6V8 and (f) BZX55C9V1, plotted at two temperature levels.

\subsubsection{Negative Zener Diode Clippers}

The transfer characteristics graph of the negative Zener diode clippers, plotted at two temperature levels of $300 \mathrm{~K}$ and 93 $\mathrm{K}$, was shown in Figure 7 . When the positive peak of the input signal is limited by Zener action and the negative voltage is limited to -0.60 Volt $\left(-\mathrm{V}_{\gamma}\right)$, measured at $300 \mathrm{~K}$, it is shown to be limited or clipped at -1.20 Volts, measured at $93 \mathrm{~K}$, for Zener diode of type BZV86-1V4 as an example (Figure 7a).

During the positive half cycle of the 9.0 Volts peak input signal, the Zener diodes is reversed biased. The Zener diode clipper will limit the positive half cycle of a signal to its reverse breakdown voltages as shown in Figure 7. For example, and from Figure $7 \mathrm{a}$, if the output waveform tries to exceed the +1.40 Volts limit at room temperature, the Zener diode will "clip-off" the excess voltage from the input producing a waveform with a flat top still keeping the output constant at +1.40 Volts $\left(\mathrm{V}_{\mathrm{z}}\right)$. On the other hand, at $93 \mathrm{~K}$, the output waveforms were shown to be clipped at +2.0 Volts.

The input-output relation of the negative parallel Zener diode clipper circuit is obtained as (Figure 7) [1]:

$$
\begin{array}{ll}
v_{0}=-v_{\gamma} & \text { for } \quad V_{i}<-v_{\gamma}, \\
V=V_{i} & \text { for }-v_{\gamma}<V_{i}<v_{Z}, \\
b=V_{Z} & \text { for } \quad V_{i}>v_{Z},
\end{array}
$$

$D_{Z}$ conducts

$D_{Z}$ is open 


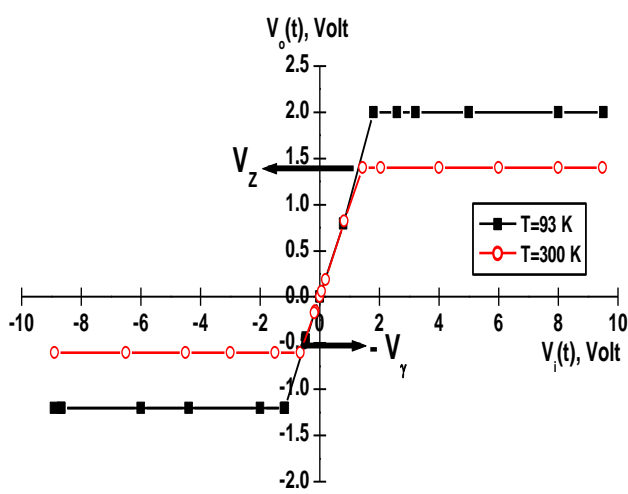

(a)

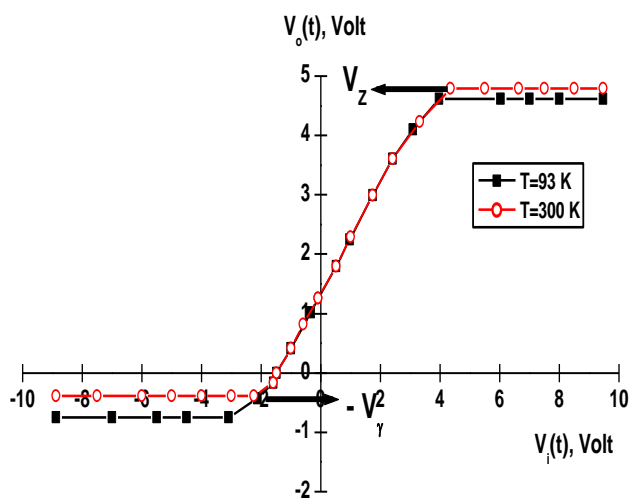

(c)

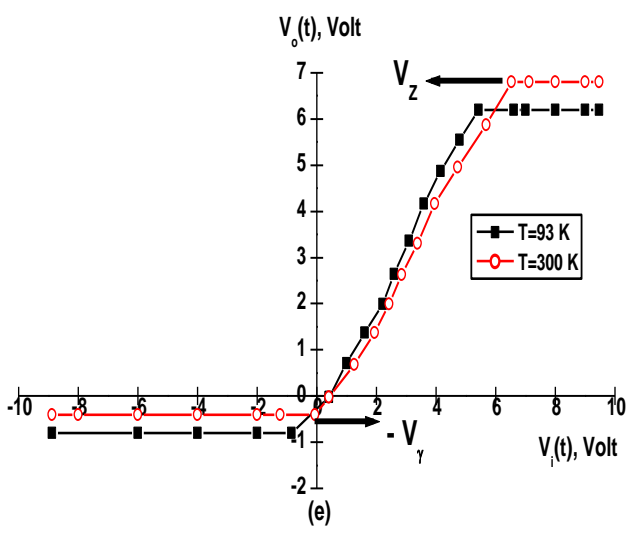

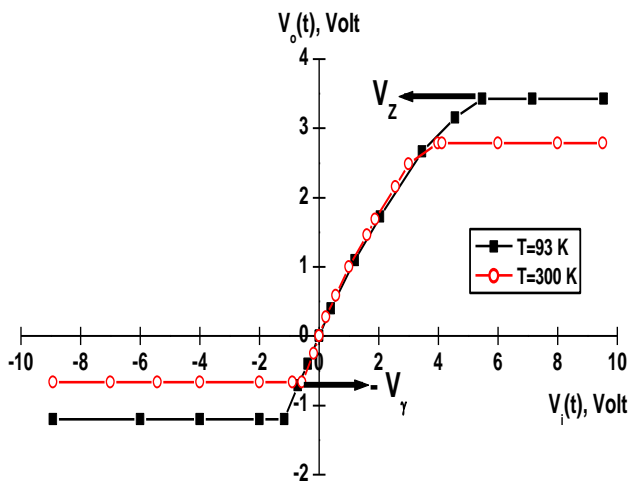

(b)

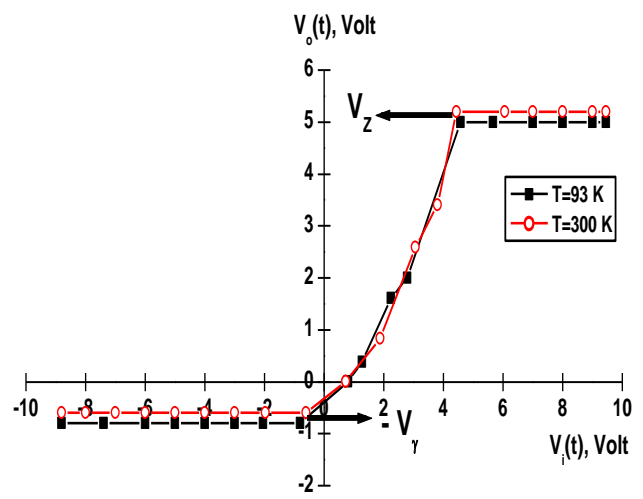

(d)

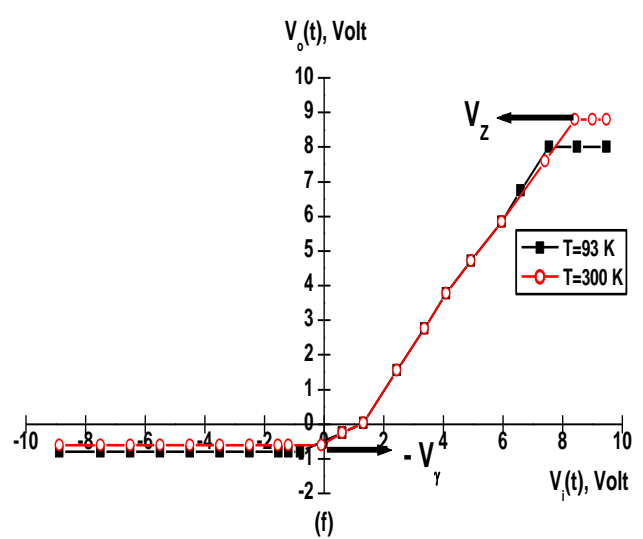

Fig 7: Transfer characteristic graphs of unsymmetrical negative clippers based on different Zener diodes: (a) BZV86-1V4, (b) BZX83-C3V6, (c) BZX79-C4V7, (d) BZX79-C5V6, (e) BZX83-C6V8 and (f) BZX55C9V1, plotted at two temperature levels.

\subsubsection{Double Ended Clippers}

The paper was extended to include the double ended clippers. In this concern, Figure 8 shows transfer function of the proposed clipper circuit (Figure 1c), plotted at two different temperature levels of $300 \mathrm{~K}$ and $93 \mathrm{~K}$ (for simplicity). When the input voltage is positive and exceeds $\left(V_{Z}+V_{\gamma}\right)$ Volt, the diodes conduct, with the $D_{1}$ forward biased (and thus with a voltage drop $\left(\mathrm{V}_{\gamma}\right)$ of 0.60 Volt) and the $\mathrm{D}_{2}$ reverse biased and in breakdown mode. If the input voltage increases, the output is held at $\left(V_{z}+V_{\gamma}\right)$ Volt. Similarly, if the input is more negative than $-\left(V_{z}+V_{\gamma}\right)$ Volt, the $D_{2}$ diode is forward biased with 0.60 Volt drop and the $D_{1}$ is reverse biased with a voltage drop of $V_{z}$. Thus, the output can never be less than $-\left(V_{Z}+V_{\gamma}\right)$ Volts or greater than $+\left(V_{Z}+V_{\gamma}\right)$ Volts [18]. 
The transfer function (Figure 8) of the proposed double ended clipper has an output voltage $V_{\circ}$ which is identical to its input so long as the input voltage in amplitude is below the breakdown voltage of one of the Zener diodes plus the forward voltage of the other Zener diode [8]. For example, as shown in (Figure 8a), where the Zener diodes are selected as being BZV86-1V4, the output voltage of the clipper circuit ranges between -1.90 Volts and +2.05 Volts, measured at room temperature, and -3.16 Volts and +3.22 Volts, measured at cryogenic level $(93 \mathrm{~K})$. If other Zener diodes are selected, as shown in (Figure 8), the clipping levels are different and correspond to the sum of the forward and reverse voltages associated with the selected Zener diodes.
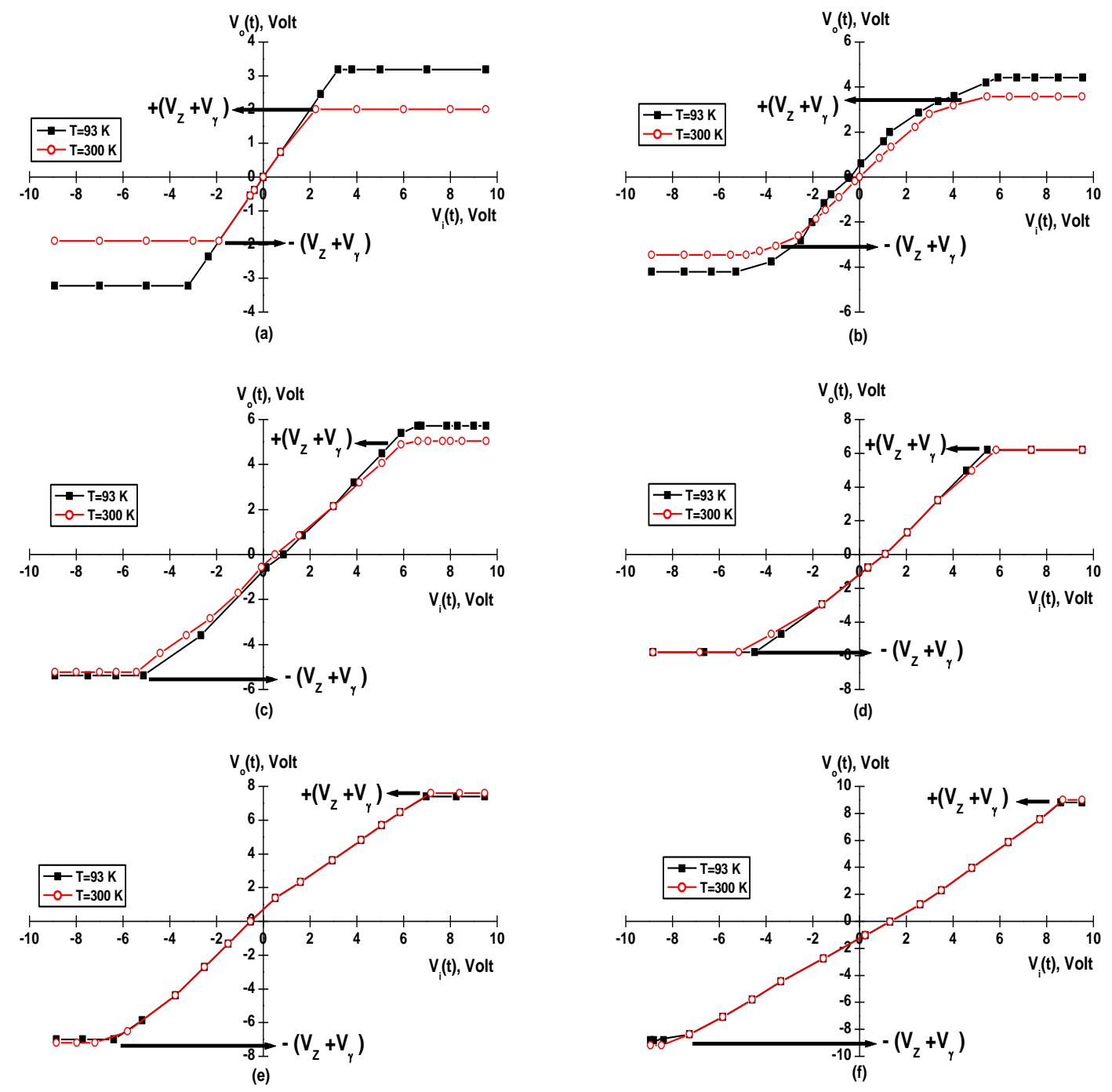

Fig 8: Transfer characteristic graphs of symmetrical Zener double ended clipper based on different Zener diodes (a) BZV86-1V4, (b) BZX83-C3V6, (c) BZX79-C4V7, (d) BZX79-C5V6, (e) BZX83-C6V8 and (f) BZX55C9V1, plotted at two temperature levels.

\section{CONCLUSIONS}

As clipper circuit is of great interest and importance in a wide number of modern electronic circuits, where it is normally employed as an entrance protective circuit to avoid voltage peaks. So, the present paper was interested in the performance, analysis, and operation of some clipper circuits based on Zener diodes. As well, the paper showed a detailed study about the effects of low temperatures levels 


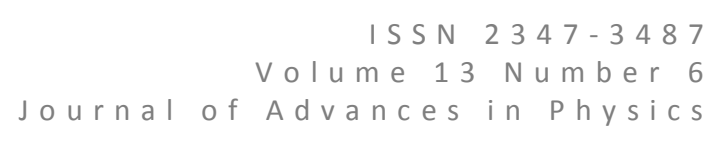

\section{REFERENCES}

1. Prakash Rao 2006 Pulse and Digital Circuits, Chapter 3, India: Tata McGraw-Hill Publishing Company Limited;.

2. Ui Ho Song 1997 Clipper Circuit for Clipping an Upper or Lower Portion of a Unidirectional Sinusoidal Voltage Signal, U.S. Patent 5,689,204, Samsung Electro-Mechanics Co., Ltd.

3. Bhaskar Y., et al., "Development of Virtual Experiment on Clippping Circuits", Intr. Journal of Computer Science and Network [IJCSN], 2012, 1(3).

4. N. P. Deshpande 2007 Electron Devices \& Circuits - Principles and Applications, India: Tata McGraw-Hill Education;

5. Markus Brunk, Ansgar J"ungel, 2008 Heating of Semiconductor Devices in Electric Circuits, 7th Intr. Conf. on Sci. Computing in Electrical Eng. SCEE, Helsinki Univ. of Technology, Espoo, Finland. Sept. 28 - Oct. 3.

6. A. Z. Rakhmatov, D. A. Petrov, A. V. Karimov, D. M. Yodgorova, O. A. Abdulkhaev, "Influence of neutron radiation on breakdown voltage of silicon voltage limiter", Radio-electronics and Commu. Sys. 2012; 55(7): pp. 332-334.

7. Robert Diffenderfer, 2005 Electronic Devices: Systems and Applications Chapter 3. USA: Thomas Delmar Learning.

8. Eugene R. Schlesinger, 1979 Circuit Having Adjustable Clipping Level", U.S. Patent 4, 138, 612, The PerkinElmer Corporation, Norwalk, Conn., Appl., United States Patent.

9. W. Abdel Basit, S. M. El-Ghanam, A. M. Abd El-Maksood and F. A. S. Soliman, Performance of shunt voltage regulators based on Zener diodes at cryogenic temperatures", NET journals: Physical Sciences Research International. February 2013; 1(1): pp. 15-24.

10. Renesas Electronics Corporation, "Old Company Name in Catalogs and Other Documents", April 1 st, 2010. Available from: http://documentation.renesas.com/doc/products/diode/rej27g0012_zener.pdf.

11. Edwd H. Hellen, 2003 "Verifying the Diode-Capacitor Circuit Voltage Decay", American Association of Physics Teachers. 71(8): pp. 797-800.

12. R. Karunanithi, S. Jacob and S. Kasthurirengan, 2000 Development of Discrete Array Type Liquid Level Indicator for Cryogenic Fluids, Adv. in Cryogenic Eng.; 45 (Parts A \& B).

13. Allnutt and Jeffrey Foster, 2007 Testing and Characterization of Silicon Devices at Cryogenic Temperatures, Fac. of the Graduate School of the Univ. of Maryland, College Park, Master of Science.

14. Duy Minh Nguyen, Gontran Pâques, Nicolas Dheilly, Christophe Raynaud, Dominique Tournier, Jens Peter Konrath, et al., "Avalanche diodes with low temperature dependence in $4 \mathrm{H}-\mathrm{SiC}$ suitable for parallel protection", Trans. Tech. Publ., Switzerland, Materials Sci. Forum. 2011; 679-680: pp. 633-636.

15. Derek F. Bowers and Ali Tasdighi 1987 CMOS temperature insensitive voltage reference, U.S. Patent 4,677,369, Precision Monolithics, Inc.

16. A.P.Godse and U.A.Bakshi, 2009 Electronics Engineering, 2nd edition, Chapter 2. India: Technical Publications Pune.

17. Rashid H. Muhammad, 2016 Microelectronic Circuits: Analysis and Design, 3rd Ed., Chapter 4; USA: Cengage Learning, USA.

18. Dennis L. Eggleston, "Basic Electronics for Scientists and Engineers", Chapter 3. USA: Cambridge University Press; 2011.

\section{Author}

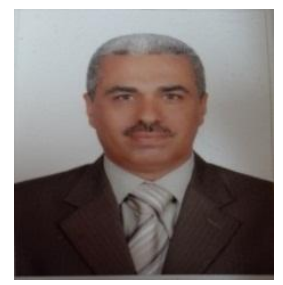

Ashraf Mosleh Abd El-Maksood, Lecturer of Electronics Engineering, Electronics Eng. Dept., Nuclear Materials Authority of Egypt. Major field of interest: Energy, Electronics, Radiation detection and computer programming and simulation.

Email:ashrafmosleh11@gmail.com 